\title{
Liver cancer with concomitant TP53 and CTNNB1 mutations: a case report
}

\author{
Juliane Friemel ${ }^{1,2}$, Markus Rechsteiner ${ }^{1}$, Marion Bawohl ${ }^{1}$, Lukas Frick ${ }^{1,4}$, Beat Müllhaupt ${ }^{3}$, Mickaël Lesurtel ${ }^{4}$ \\ and Achim Weber ${ }^{1 *}$
}

\begin{abstract}
Background: In the spectrum of molecular alterations found in hepatocellular carcinoma (HCC), somatic mutations in the WNT/ $\beta$-catenin pathway and the p53/cell cycle control pathway are among the most frequent ones. It has been suggested that both mutations occur in a mutually exclusive manner and they are used as molecular classifiers in HCC classification proposals.

Case presentation: Here, we report the case of a treatment-naïve mixed hepatocellular/cholangiocellular carcinoma (HCC/CCC) with morphological and genetic intratumor heterogeneity. Within the predominant part of the tumor with hepatocellular differentiation, a p.D32V mutation in exon 3 of the CTNNB1 gene occurred concomitantly with a TP53 intron 7/exon 8 splice site mutation.

Conclusion: Intratumor heterogeneity challenges the concept of CTNNB1 and TP53 gene mutations being mutually exclusive molecular classifiers in HCC, which has implications for HCC classification approaches.
\end{abstract}

Keywords: Hepatocellular carcinoma (HCC), Intratumor heterogeneity, CTNNB1, TP53, Next generation sequencing

\section{Background}

Hepatocellular carcinoma (HCC) is the fifths most common cancer in men and the second most common cause for cancer-related death worldwide [1]. HCC mostly develop on the background of chronic liver diseases including chronic viral hepatitis due to infection with hepatitis $\mathrm{B}$ virus (HBV), or hepatitis $\mathrm{C}$ virus $(\mathrm{HCV})$, alcohol-induced liver injury, fatty liver disease or exposure to toxic factors such as aflatoxin. The spectrum of somatic mutations related to liver carcinogenesis has been identified [2]. With marked geographic variation, TP53 and CTNNB1 represent two of the most common driver mutations in the African-Asian countries (TP53) and in the western world (CTNNB1). Several molecular classifications of $\mathrm{HCC}$ distinguish HCC with alterations in the p53/cell cycle control pathway from HCCs with alterations in the $\mathrm{WNT} / \beta$-catenin pathway, including activating mutations of the CTNNB1 oncogene, AXIN1 or

\footnotetext{
* Correspondence: achim.weber@usz.ch

${ }^{1}$ Institute of Surgical Pathology, University and University Hospital Zurich, Schmelzbergstrasse 12, 8091 Zurich, Switzerland

Full list of author information is available at the end of the article
}

$A P C[3,4]$. Mutations of TP53 and CTNNB1 are largely considered to occur in a mutually exclusive manner [5]. Phenotypical and genetic intratumor heterogeneity with variable mutational status (i.e. wild type among mutated tumor cell clones) of TP53 and CTNNB1 in different tumor regions within the same tumor is frequently found in HCC [6]. Here, we describe a de novo, hepatitis C-related combined cholangiocellular and hepatocellular carcinoma with marked intratumor heterogeneity on three levels: morphology, immunohistochemical marker profile and mutational status with 3/14 tumor regions of solely hepatocellular differentiation harboring concomitant mutations of CTNNB1 and TP53.

\section{Case presentation}

A liver tumor was detected in a 72 year old male patient with liver cirrhosis Child-Pugh Stage A, a history of type 2 diabetes and chronic hepatitis $\mathrm{C}$ virus infection $(\mathrm{HCV}$, genotype 1B), initially diagnosed 13 years ago. Liver enzymes were slightly elevated with alanine aminotransferase $89 \mathrm{U} / \mathrm{L}$ (reference: 10-50 U/L) and aspartate aminotransferase $65 \mathrm{U} / \mathrm{L}$ (reference: $<50 \mathrm{U} / \mathrm{L}$ ). The $4 \mathrm{~cm}$ tumor was detected by routine sonography and removed by laparoscopic liver segment resection. 
Morphological analysis, immunohistochemistry and multiregional, next generation sequencing (NGS) was applied on representative tumor sections as described [6]. Table 1 and Fig. 1 illustrate histopathological and molecular findings in 14 individual tumor areas, which were grouped into three tumor regions (A, B and C) according to their predominant morphological and molecular characteristics. In summary, a multinodular, combined hepatocellular/cholangiocellular carcinoma, tumor stage T1 grade 2-3, was diagnosed. Intratumoral heterogeneous expression of five liver cell markers (CK7, CK19, glutamine synthetase, $\mathrm{p} 53, \beta$-catenin) was detected including a double positivity for glutamine synthetase, nuclear $\beta$ catenin and p53 in tumor region $\mathrm{A}$.

Next generation sequencing was performed with a minimum coverage of 1329 (SD \pm 725 ) reads per amplicon of every single tumor area. Sequencing results yielded a p.D32V (c.363 A > T) mutation and a TP53 ivs8-1 (c.783-1 G > A) splice site mutation in tumor region A. Comparing mutated allele frequencies, CTNNB1 and TP53 gene copies showed a similar range of both frequencies in area A1-3 (Fig. 1). All mutated and wild type tumor areas additionally displayed a SNP of exon 7 (rs17880604). Collectively, morphological and immunohistochemical findings together with sequencing results demonstrated that a tumor subclone with hepatocellular differentiation had concomitant CTNNB1 and TP53 gene mutations.

To date, after a follow-up time of 12 months, the patient had a local recurrence of a liver tumor which was inoperable and therefore treated by transarterial chemoembolization (TACE).

\section{Conclusion}

In this case of a $\mathrm{HCV}$ infection -related liver cancer, a CTNNB1/TP53 double mutation was detected in a tumor region of hepatocellular differentiation, among TP53 and CTNNB1 wild type tumor areas. The analysis of mutated allele frequencies using next generation sequencing techniques corroborates that the double mutation is located in the same tumor cell population. To our knowledge, this is the first detailed description of a CTNNB1/TP53 double mutation in a single liver cancer lesion. TP53 and CTNNB1 both are molecular classifiers for hepatocellular carcinoma. For instance, in the transcriptome-analysis based classification proposal by Boyault et al. [4], six HCC subgroups are distinguished: two groups are characterized by TP53 and two independent groups by CTNNB1 alterations. A study by Laurent-Puig et al. [5] on genetic alterations in hepatocarcinogenesis describes TP53 and CTNNB1 mutations as mutually exclusive. In agreement, a study by Tornesello et al. [7] records mutations of the two driver genes as being mutually exclusive.

CTNNB1 mutations are reported to be associated with hepatitis $C$ infections [8]. TP53 point mutations frequently are reported to occur specifically at codon 249 after aflatoxin exposition. The frequency and the causal link between TP53 and CTNNB1 mutations in HCC have not been systematically investigated. A study by Ötztürk et al. [9] on HCC cell lines provides evidence that inactivation of TP53 could cause aberrant nuclear $\beta$-catenin accumulation, suggesting a link between the two genes. In the presented case study, the CTNNB1 mutation affected the GSK-3 $\beta$ phosphorylation site [10] which argues for a $\beta$-catenin accumulation independent

Table 1 Morphology, immunohistochemistry and mutational status of individual tumor areas

\begin{tabular}{|c|c|c|c|c|c|c|c|c|c|c|c|c|c|c|c|}
\hline & Area & A1 & $\mathrm{A} 2$ & A3 & $B$ & $\mathrm{C} 1$ & C2 & C3 & C4 & C5 & C6 & $C 7$ & C8 & C9 & C10 \\
\hline & Size in $\mathrm{mm}^{2}$ & 7.8 & 5.54 & 31.3 & 65 & 73.2 & 16.5 & 34.5 & 82.2 & 20.2 & 18.9 & 17.4 & 28.5 & 1.94 & $\overline{17.5}$ \\
\hline \multirow[t]{6}{*}{ Morphology } & Solid & + & + & + & & + & + & + & & + & + & & + & & \\
\hline & Glandular (cholangiocellular) & & & & + & & & & & & & & & & \\
\hline & Trabecular & & & & & & & + & + & & & + & & + & + \\
\hline & Clear cell change & & + & & & + & & & & & & & & & + \\
\hline & Fatty change & & & & & & & & & & & + & & & \\
\hline & CK19 & & & & $>50 \%$ & & $>50 \%$ & & & & & & & & \\
\hline \multirow[t]{4}{*}{ IC } & CK7 & & & & $70 \%$ & SC & $50 \%$ & $20 \%$ & SC & $40 \%$ & SC & & SC & & $50 \%$ \\
\hline & glutamine synthetase & + & + & + & & & & & & & & & & & \\
\hline & $\beta$-catenin nuclear & + & + & + & & & & & & & & & & & \\
\hline & p53 & + & + & + & & & & & & & & & & & \\
\hline \multirow[t]{4}{*}{ Mutation } & CTNNB1 sanger seq & D32 & D32 & wt & wt & wt & wt & wt & wt & wt & wt & wt & wt & wt & wt \\
\hline & CTNNB1 deep seq & D32 & D32 & $\mathrm{D} 32^{\mathrm{a}}$ & wt & wt & wt & wt & wt & wt & wt & wt & wt & wt & wt \\
\hline & TP53 sanger seq & IVS8-1 & IVS8-1 & wt & wt & wt & wt & wt & wt & wt & wt & wt & wt & wt & wt \\
\hline & TP53 deep seq & IVS8-1 & IVS8-1 & IVS8-1 ${ }^{a}$ & wt & wt & wt & wt & wt & wt & wt & wt & wt & wt & wt \\
\hline
\end{tabular}

sanger seq sanger sequencing, deep seq deep sequencing (NGS), wt wild type, SC single cells positive 


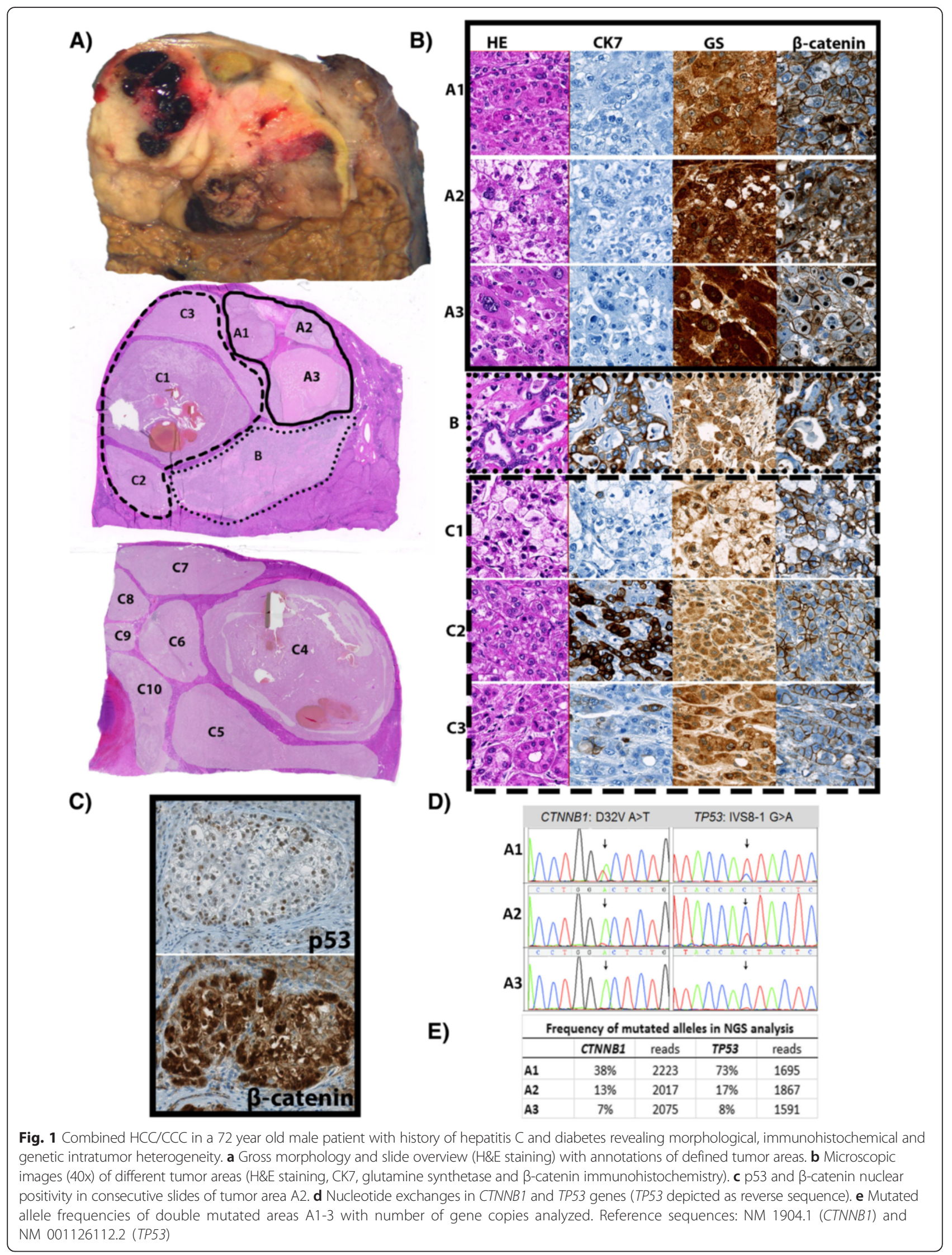


from the TP53 mutation. The detected TP53 mutation affects a splice site of exon 8. Although splice sites in TP53 are not typical mutation sites, there is evidence that TP53 splicing mutations lead to exon dropping indicating biological relevance [11]. Furthermore, the nuclear accumulation of the dysfunctional p53 protein in immunohistochemical analysis found in this case supports a functional significance of this splice site mutation. As has been reported, wild type p53 is rapidly degraded while mutations lead to nuclear accumulation of the p53 protein $[12,13]$.

In summary, the molecular, immunohistochemical and morphological diversity in the presented case indicates a high level of intratumor heterogeneity and challenges the concept that TP53 and CTNNB1 are mutually exclusive driver alterations in HCC. Distinct parts of the tumor reveal multinodularity, and differ with respect to their biomarker expression and mutational status, indicative of distinct tumor subpopulations [14]. This finding illustrates the challenge to molecularly characterize individual HCC. Routine pathological analysis is based on testing a small piece of a tumor, assuming that it represents the whole tumor. When analyzing distinct pathways of hepatocarcinogenesis such as the $\mathrm{WNT} / \beta$-catenin pathway as a potential therapeutic target in HCC [15], it will be pivotal in the future to also take into account the level of intratumor heterogeneity.

\section{Abbreviations}

CCC, cholangiocellular carcinoma; CTNNB1, Catenin $\beta$ 1; HCC, hepatocellular carcinoma; TACE, transarterial chemoembolization; TP53, tumor protein 53

\section{Acknowledgements}

We gratefully thank the patient and family members for allowing us to report this case.

\section{Funding}

This work was supported by the following grants: A grant from the Theiler-Haag Stiftung, Zurich to AW and LF. Grants from the Krebsliga Schweiz (Oncosuisse) and from the "Kurt and Senta Herrmann Stiftung", Vaduz, Lichtenstein to AW.

\section{Availability of data and materials}

Not applicable.

\section{Authors' contributions}

Conception: AW. Methods: JF, LF, MR, MB. Patient care and surgery: ML, BM. Writing of the manuscript: JF and AW. Critical revision of the manuscript: LF, ML, BM, AW, JF, MB. All authors read and approved the final manuscript.

\section{Authors' information}

AW is Professor for Experimental and Molecular Pathology at the University (UZH) and University-Hospital (USZ) Zurich.

\section{Competing interests}

The authors declare that they have no competing interests.

\section{Consent for publication}

Written informed consent was obtained from the patient for publication of this case report and any accompanying images. A copy of the written consent is available for review by the Editor of this journal.
Ethics approval and consent to participate

Ethical approval for the study was given by the local ethics commitee (StV 26-2005 and KEK-ZH-Nr. 2013-0382).

\section{Author details}

${ }^{1}$ Institute of Surgical Pathology, University and University Hospital Zurich, Schmelzbergstrasse 12, 8091 Zurich, Switzerland. ${ }^{2}$ Leibniz Institute for Prevention Research and Epidemiology (BIPS), Bremen, Germany. ${ }^{3}$ Clinics of Hepatology and Gastroenterology, University and University Hospital Zurich, Zurich, Switzerland. ${ }^{4}$ Swiss Hepato-Pancreato-Biliary Center, Department of Digestive and Transplant Surgery, University Hospital of Zurich, Zurich, Switzerland.

Received: 14 December 2015 Accepted: 21 May 2016

Published online: 01 June 2016

\section{References}

1. Ferlay J, Soerjomataram I, Dikshit R, Eser S, Mathers C, Rebelo M, Parkin DM, Forman D, Bray F. Cancer incidence and mortality worldwide: sources, methods and major patterns in GLOBOCAN 2012. Int J Cancer. 2015;136(5): E359-86.

2. Nault JC, Mallet M, Pilati C, Calderaro J, Bioulac-Sage P, Laurent C, Laurent A, Cherqui D, Balabaud C, Zucman-Rossi J. High frequency of telomerase reverse-transcriptase promoter somatic mutations in hepatocellular carcinoma and preneoplastic lesions. Nat Commun. 2013;4:2218.

3. Guichard C, Amaddeo G, Imbeaud S, Ladeiro Y, Pelletier L, Maad IB, Calderaro J, Bioulac-Sage P, Letexier M, Degos F, et al. Integrated analysis of somatic mutations and focal copy-number changes identifies key genes and pathways in hepatocellular carcinoma. Nat Genet. 2012;44(6):694-8.

4. Boyault S, Rickman DS, de Reynies A, Balabaud C, Rebouissou S, Jeannot E, Herault A, Saric J, Belghiti J, Franco D, et al. Transcriptome classification of $\mathrm{HCC}$ is related to gene alterations and to new therapeutic targets. Hepatology. 2007:45(1):42-52.

5. Laurent-Puig P, Legoix P, Bluteau O, Belghiti J, Franco D, Binot F, Monges $G$, Thomas G, Bioulac-Sage P, Zucman-Rossi J. Genetic alterations associated with hepatocellular carcinomas define distinct pathways of hepatocarcinogenesis. Gastroenterology, 2001:120(7):1763-73.

6. Friemel J, Rechsteiner M, Frick L, Bohm F, Struckmann K, Egger M, Moch H, Heikenwalder M, Weber A. Intratumor heterogeneity in hepatocellular carcinoma. Clin Cancer Res. 2015;21(8):1951-61.

7. Tornesello ML, Buonaguro L, Tatangelo F, Botti G, Izzo F, Buonaguro FM. Mutations in TP53, CTNNB1 and PIK3CA genes in hepatocellular carcinoma associated with hepatitis B and hepatitis C virus infections. Genomics. 2013; 102(2):74-83.

8. Huang H, Fujii H, Sankila A, Mahler-Araujo BM, Matsuda M, Cathomas G, Ohgaki $\mathrm{H}$. Beta-catenin mutations are frequent in human hepatocellular carcinomas associated with hepatitis C virus infection. Am J Pathol. 1999; 155(6):1795-801.

9. Cagatay T, Ozturk M. P53 mutation as a source of aberrant beta-catenin accumulation in cancer cells. Oncogene. 2002;21(52):7971-80.

10. de La Coste A, Romagnolo B, Billuart P, Renard CA, Buendia MA, Soubrane $\mathrm{O}$, Fabre M, Chelly J, Beldjord C, Kahn A, et al. Somatic mutations of the beta-catenin gene are frequent in mouse and human hepatocellular carcinomas. Proc Natl Acad Sci U S A. 1998:95(15):8847-51.

11. Lai MY, Chang HC, Li HP, Ku CK, Chen PJ, Sheu JC, Huang GT, Lee PH, Chen DS. Splicing mutations of the p53 gene in human hepatocellular carcinoma. Cancer Res. 1993;53(7):1653-6.

12. Hsu HC, Tseng HJ, Lai PL, Lee PH, Peng SY. Expression of p53 gene in 184 unifocal hepatocellular carcinomas: association with tumor growth and invasiveness. Cancer Res. 1993;53(19):4691-4.

13. Chen GG, Merchant IL, Lai PB, Ho RL, Hu X, Okada M, Huang SF, Chui AK, Law DJ, Li YG, et al. Mutation of p53 in recurrent hepatocellular carcinoma and its association with the expression of ZBP-89. Am J Pathol. 2003;162(6): 1823-9.

14. Kanai T, Hirohashi S, Upton MP, Noguchi M, Kishi K, Makuuchi M, Yamasaki $\mathrm{S}$, Hasegawa H, Takayasu K, Moriyama N, et al. Pathology of small hepatocellular carcinoma. A proposal for a new gross classification. Cancer. 1987:60(4):810-9.

15. Park JY, Park WS, Nam SW, Kim SY, Lee SH, Yoo NJ, Lee JY, Park CK. Mutations of beta-catenin and AXIN I genes are a late event in human hepatocellular carcinogenesis. Liver Int. 2005;25(1):70-6. 